\title{
Dynamical formation of horizons in recoiling D-branes
}

\author{
John Ellis \\ Theory Division, CERN, CH-1211 Geneva 23, Switzerland
}

N. E. Mavromatos

Theoretical Physics Group, Department of Physics, King's College London, Strand, London WC2R 2LS, United Kingdom

D. V. Nanopoulos

Department of Physics, Texas A \& M University, College Station, Texas 77843-4242,

Astroparticle Physics Group, Houston Advanced Research Center (HARC), Mitchell Campus, Woodlands, Texas 77381, and Academy of Athens, Chair of Theoretical Physics, Division of Natural Sciences, 28 Panepistimiou Avenue, Athens 10679, Greece

(Received 9 June 2000; published 22 September 2000)

\begin{abstract}
A toy calculation of string or D-particle interactions within a world-sheet approach indicates that quantum recoil effects-reflecting the gravitational back reaction on space-time foam due to the propagation of energetic particles-induces the appearance of a microscopic event horizon, or "bubble," inside which stable matter can exist. The scattering event causes this horizon to expand, but we expect quantum effects to cause it to contract again, in a "bounce" solution. Within such "bubbles," massless matter propagates with an effective velocity that is less than the velocity of light in vacuo, which may lead to observable violations of Lorentz symmetry that may be tested experimentally. The conformal invariance conditions in the interior geometry of the bubbles select preferentially 3 for the number of the spatial dimensions, corresponding to a consistent formulation of the interaction of D3-branes with recoiling D particles, which are allowed to fluctuate independently only on the D3-brane hypersurface.
\end{abstract}

PACS number(s): 04.50.+h, 04.62.+v, 98.80.Cq

\section{INTRODUCTION}

The discovery of D-branes [1] has revolutionized the study of black-hole physics. Now one has quasi-realistic string models of black holes in different dimensions, which one can use to study profound issues concerning the reconciliation of general relativity and quantum mechanics. A key breakthough was the demonstration that the entropy of a stringy black hole corresponds to the number of its distinct quantum states $[2,3]$. Thus D-branes offer the prospect of accounting exactly for the flow of information in processes involving particles and black holes. However, it is not immediately apparent whether an observer will perceive information to be lost in any given particle-D-brane interaction: the answer depends on whether she is able to recover all the information transferred from the scattering particle to the recoiling black hole. It is important to address this issue at both the macroscopic and microscopic levels, where the answers may differ. In the case of a macroscopic black hole, it is difficult to see how in practice all the quantum information may be recovered without a complete set of observations of the emitted Hawking radiation [4]. However, even if this is possible in principle, the problem of the microscopic "end game" that terminates the Hawking evaporation process is unsolved, in our view.

It may be useful to recall one of the intuitive ways of formulating the information loss in the process of Hawking radiation from a macroscopic black hole, whose stringy analogue we study in this paper. Consider the quantummechanical creation of a pure-state particle pair $|A, B\rangle$ close to the (classical) black-hole horizon of such a macroscopic black hole. One can then envisage that particle $B$ falls inside this horizon, while particle $A$ escapes as Hawking radiation. The quantum state of the particle $B$ is apparently unobservable, and hence information is apparently lost. One may represent the corresponding quantum process as

$$
|A, B\rangle+|B H\rangle \rightarrow\left|B H+B_{i}\right\rangle+\left|A_{i}\right\rangle .
$$

The observable subset $A_{i}$ of the final state can only be represented by a density matrix

$$
\rho_{A} \equiv \Sigma_{i}\left|A_{i}\right\rangle\left\langle A_{i}\right|,
$$

and it appears that the pure state $|A, B\rangle$ evolves into a mixed density matrix $\rho_{A}$, representing (almost) a thermal state.

This argument is very naive, and one would like to formulate a more precise treatment of this process at the microscopic level, suitable for describing space-time foam [5]. The purpose of this paper is to take a step towards this goal using a stringy treatment [6-8] of the interaction between closedstring particle "probes" and D-brane black holes. We have developed previously an approach capable of accommodating the recoil of a D-brane black hole struck by a closedstring "probe," including also quantum effects associated with higher-genus contributions to the string path integral. We have shown explicitly $[9,7]$ how the loss of information to the recoiling D-brane (assuming that it is unobserved) leads to information loss, for both the scattered particle and also any spectator particle. This information loss can be related to a change in the background metric following the scattering event, which can be regarded as creating an Unruh-like "thermal" state.

In this paper, we take this line of argument a step further by demonstrating that closed-string particle or D-brane scat- 
tering leads in general to the formation of a microscopic event horizon, within which string particles may be trapped. The scattering event causes an expansion of this horizon, which is eventually halted and reversed by Hawking radiation [4]. Thus we have a microscopic stringy realization of the process (2) discussed intuitively above.

A peculiarity of this approach is that the conformal invariance conditions select preferentially backgrounds with three spatial dimensions. This leads to a consistent formulation of the interaction of D3-branes with recoiling D particles, which are allowed to fluctuate independently only on the D3-brane hypersurface.

\section{FORMULATION OF D-BRANE RECOIL}

As discussed in Refs. [6-8], the recoil of a D-brane string soliton after interaction with a closed-string state is characterized by a $\sigma$ model on the string world sheet $\Sigma$, which is deformed by a pair of logarithmic operators [10]

$$
C_{\epsilon}^{I}=\epsilon \Theta_{\epsilon}\left(X^{I}\right), \quad D_{\epsilon}^{I}=X^{I} \Theta_{\epsilon}\left(X^{I}\right), \quad I \in\{0, \ldots, 3\},
$$

defined on the boundary $\partial \Sigma$ of the string world sheet. Here $X^{I}, \quad I \in\{0, \ldots, p\}$, obey Neumann boundary conditions on $\Sigma$, and denote the D-brane coordinates, while $\epsilon \rightarrow 0^{+}$is a regulating parameter and $\Theta_{\epsilon}\left(X^{I}\right)$ is a regularized Heaviside step function. The remaining $y^{i}, \quad i \in\{p+1, \ldots, 9\}$, in Eq. (3) denote the transverse bulk directions. For reasons of convergence of the world-sheet path integral, we take the spacetime $\left\{X^{I}, y^{i}\right\}$ to have Euclidean signature.

In the case of D particles [6-8], the index $I$ takes the value 0 only, in which case the operators (3) act as deformations of the conformal field theory on the world sheet. The operator

$$
u_{i} \int_{\partial \Sigma} \partial_{n} X^{i} D_{\epsilon}
$$

describes the movement of the D-brane induced by the scattering, where $u_{i}$ is its recoil velocity, and $y_{i} \int_{\partial \Sigma} \partial_{n} X^{i} C_{\epsilon}$ describes quantum fluctuations in the initial position $y_{i}$ of the $\mathrm{D}$ particle. It has been shown rigorously [8] that the logarithmic conformal algebra ensures energy-momentum conservation during the recoil process:

$$
u_{i}=\left(k_{i}^{1}+k_{i}^{2}\right) / M_{D},
$$

where $k^{1}\left(k^{2}\right)$ is the momentum of the propagating closed string state before (after) the recoil, and $M_{D}=1 /\left(l_{s} g_{s}\right)$ is the mass of the D-brane, where $g_{s}$ is the string coupling, which is assumed here to be weak enough to ensure that the D-brane is very massive, and $l_{s}$ is the string length.

The second member of the logarithmic pair of $\sigma$-model deformations is

$$
y_{i} \int_{\partial \Sigma} \partial_{n} X^{i} C_{\epsilon}
$$

where, in order to realize the logarithmic algebra between the operators $C$ and $\mathrm{D}$, one uses as a regulating parameter [6]

$$
\epsilon^{-2} \sim \ln [L / a] \equiv \Lambda
$$

where $L(a)$ is an infrared (ultraviolet) world-sheet cutoff. The recoil operators (6) are relevant, in the sense of the renormalization group for the world-sheet field theory, having small conformal dimensions $\Delta_{\epsilon}=-\epsilon^{2} / 2$. Thus the $\sigma$ model perturbed by these operators is not conformal for $\epsilon \neq 0$, and the theory requires Liouville dressing $[11,12,7]$. The consistency of this approach is supported by the abovementioned proof of momentum conservation during the scattering process [8].

As discussed in [7,9], the deformations (3) create a local distortion of the space-time surrounding the recoiling D-brane, which may also be determined using the method of Liouville dressing. In [7,9] we concentrated on describing the resulting space-time in the case when a D particle defect embedded in a $D$-dimensional space-time recoils after the scattering of a closed string. To leading order in the recoil velocity $u_{i}$ of the $\mathrm{D}$ particle, the resulting space-time was found, for times $t \gg 0$ long after the scattering event at $t=0$, to be equivalent to a Rindler wedge, with apparent " acceleration" $\epsilon u_{i}$ [9], where $\epsilon$ is defined above Eq. (7). For times $t<0$, the space-time is flat Minkowski. ${ }^{1}$

This situation is easily generalized to $\mathrm{D} p$-branes [13]. The folding and recoil deformations of the $\mathrm{D} p$-brane (6) are relevant deformations, with anomalous dimension $-\epsilon^{2} / 2$, which disturbs the conformal invariance of the world-sheet $\sigma$ model, and restoration of conformal invariance again requires Liouville dressing $[11,12,7]$, as discussed above. To determine the effect of such dressing on the space-time geometry, it is essential to write [7] the boundary recoil deformations as bulk world-sheet deformations

$$
\int_{\partial \Sigma} \bar{g}_{I z} x \Theta_{\epsilon}(x) \partial_{n} z=\int_{\Sigma} \partial_{\alpha}\left[\bar{g}_{I z} x \Theta_{\epsilon}(x) \partial^{\alpha} z\right],
$$

where the $\bar{g}_{I z}$ denote renormalized folding and recoil couplings [8]. Such couplings are marginal on a flat world sheet, and the operators (8) are marginal also on a curved world sheet, provided [12] one dresses the (bulk) integrand by multiplying it by a factor $e^{\alpha_{I i} \phi}$, where $\phi$ is the Liouville field and $\alpha_{I i}$ is the gravitational conformal dimension. This is related to the flat-world-sheet anomalous dimension $-\epsilon^{2} / 2$ of the recoil operator, viewed as a bulk world-sheet deformation by [12]

$$
\alpha_{I i}=-\frac{Q_{b}}{2}+\sqrt{\frac{Q_{b}^{2}}{4}+\frac{\epsilon^{2}}{2}}
$$

where $Q_{b}$ is the central-charge deficit of the bulk world-sheet theory. In the recoil problem at hand, as discussed in [9],

\footnotetext{
${ }^{1}$ There is hence a discontinuity at $t=0$, which leads to particle production and decoherence for a low-energy spectator field theory observer who performs local scattering experiments long after the scattering, and far away from the location of the collision of the closed string with the D particle [9].
} 


$$
Q_{b}^{2} \sim \epsilon^{4} / g_{s}^{2}>0
$$

for weak folding deformations $g_{I i}$, and hence one is confronted with a supercritical Liouville theory. This implies a Minkowskian-signature Liouville-field kinetic term in the respective $\sigma$ model [14], which prompts one to interpret the Liouville field as a time-like target field.

There are two approaches which one can follow at this point. In the first of them [15], this time is considered as a second time coordinate [16], which is independent of the (Euclideanized) $X^{0}$. The presence of this second 'time' does not affect physical observables, which are defined for appropriate slices with fixed Liouville coordinate, e.g., $\phi \rightarrow \infty$ or equivalently $\epsilon \rightarrow 0$. From the expression (10) we conclude [cf. Eq. (9)] that $\alpha_{I i} \sim \epsilon$ to leading order in perturbation theory in $\epsilon$, to which we restrict ourselves here. In the second approach [16], which we shall mainly follow here, the (Minkowskian) Liouville field $\phi$ is identified with the (initially Euclidean) coordinate $X^{0}$, and hence one is no longer considering constant Liouville field slices. In this approach, however, one still identifies $\epsilon^{-2}$ with the target time, which in turn implies that the perturbative world-sheet approach is valid, provided one works with sufficiently large times $t$, i.e. small $\epsilon^{2}$.

We next remark [7] that the $X^{I}$-dependent field operators $\Theta_{\epsilon}\left(X^{I}\right)$ scale as follows with $\epsilon$ : $\Theta_{\epsilon}\left(X^{I}\right) \sim e^{-\epsilon X^{I}} \Theta\left(X^{I}\right)$, where $\Theta\left(X^{I}\right)$ is a Heaviside step function without any field content, evaluated in the limit $\epsilon \rightarrow 0^{+}$. The bulk deformations, therefore, yield the following $\sigma$-model terms:

$$
\frac{1}{4 \pi l_{s}^{2}} \int_{\Sigma} \sum_{I=0}^{3}\left(\epsilon^{2} \bar{g}_{I i}^{C}+\epsilon \bar{g}_{I i} X^{I}\right) e^{\epsilon\left(\phi_{(0)}-X_{(0)}^{I}\right)} \Theta\left(X_{(0)}^{I}\right) \partial_{\alpha} \phi \partial^{\alpha} y_{i}
$$

where the subscripts $(0)$ denote world-sheet zero modes, and $\bar{g}_{0 i}^{C}=y_{i}$.

Upon the interpretation of the Liouville zero mode $\phi_{(0)}$ as a (second) time-like coordinate, the deformations (11) yield metric deformations of the generalized space-time with two times. The metric components for fixed Liouville-time slices can be interpreted [7] as expressing the distortion of the space-time surrounding the recoiling D-brane soliton.

For clarity, we now drop the subscripts (0) for the rest of this paper, and we work in a region of space-time such that $\epsilon\left(\phi-X^{I}\right)$ is finite in the limit $\epsilon \rightarrow 0^{+}$. The resulting spacetime distortion is therefore described by the metric elements

$$
\begin{aligned}
G_{\phi \phi} & =-1, \quad G_{i j}=\delta_{i j}, \quad G_{I J}=\delta_{I J}, \quad G_{i I}=0, \\
G_{\phi i} & =\left(\epsilon^{2} \bar{g}_{I i}^{C}+\epsilon \bar{g}_{I i} X^{I}\right) \Theta\left(X^{I}\right), \quad i=4, \ldots, 9, \quad I=0, \ldots, 3
\end{aligned}
$$

where the index $\phi$ denotes Liouville "time," not to be confused with the Euclideanized time which is one of the $X^{I}$. To leading order in $\epsilon \bar{g}_{I i}$, we may ignore the $\epsilon^{2} \bar{g}_{I i}^{C}$ term. The presence of $\Theta\left(X^{I}\right)$ functions and the fact that we are working in the region $y_{i}>0$ indicate that the induced space-time is piecewise continuous. ${ }^{2}$ In the general recoil-folding case considered in this article, the form of the resulting patch of the surrounding space-time can be determined fully if one computes the associated curvature tensors, along the lines of [9].

We next study in more detail some physical aspects of the metric (12), restricting ourselves, for simplicity, to the case of a single Dirichlet dimension $z$ that plays the role of a bulk dimension in a setup where there are Neumann coordinates $X^{I}, \quad I=0, \ldots, 3$ parametrizing a D4- (Euclidean) brane, interpreted as our four-dimensional space-time. Upon performing the time transformation $\phi \rightarrow \phi-\frac{1}{2} \epsilon \bar{g}_{I z} X^{I} z$, the line element (12) becomes

$$
\begin{aligned}
d s^{2}= & -d \phi^{2}+\left(\delta_{I J}-\frac{1}{4} \epsilon^{2} \bar{g}_{I z} \bar{g}_{J z} z^{2}\right) d X^{I} d X^{J} \\
& +\left(1+\frac{1}{4} \epsilon^{2} \bar{g}_{I z} \bar{g}_{J z} X^{I} X^{J}\right) d z^{2}-\epsilon \bar{g}_{I z} z d X^{I} d \phi,
\end{aligned}
$$

where $\phi$ is the Liouville field which, we remind the reader, has Minkowskian signature in the case of supercritical strings that we are dealing with here.

One may now make a general coordinate transformation on the brane $X^{I}$ that diagonalizes the pertinent inducedmetric elements in Eq. (13). ${ }^{3}$ For instance, to leading order in the deformation couplings $\bar{g}_{I z} \bar{g}_{J z}$, one may redefine the $X^{I}$ coordinates by

$$
\begin{aligned}
& X^{I} \rightarrow X^{I}-\frac{\epsilon^{2}}{8} z^{2} \bar{g}_{I z} \sum_{J \pm I} \bar{g}_{J z} X^{J}, \\
& z \rightarrow z\left(1+\frac{\epsilon^{2}}{8} \sum_{I \neq J} \bar{g}_{I z} \bar{g}_{J z} X^{I} X^{J}\right)
\end{aligned}
$$

which leaves only diagonal elements of the metric tensor on the (redefined) hyperplane $X^{I}$. In this case, the metric becomes, to leading order in $g_{I z}^{2}$ and in the case where $\epsilon \bar{g}_{I z} z$ $\ll 1$,

$$
\begin{aligned}
d s^{2}= & -d \phi^{2}+\left(1-\alpha^{2} z^{2}\right)\left(d X^{I}\right)^{2}+\left[1+\alpha^{2}\left(X^{I}\right)^{2}\right] d z^{2} \\
& -\epsilon \bar{g}_{I z} z d X^{I} d \phi \\
\alpha= & \frac{1}{2} \epsilon \bar{g}_{I z} \sim g_{s}\left|\Delta P_{z}\right| / M_{s}
\end{aligned}
$$

where the last expression is a reminder that one can express the parameter $\alpha$ (in the limit $\epsilon \rightarrow 0^{+}$) in terms of the (recoil) momentum transfer $\Delta P_{z}$ along the bulk direction.

\footnotetext{
${ }^{2}$ The important implications for non-thermal particle production and decoherence for a spectator low-energy field theory in such space-times were discussed in [9,7], where the D-particle recoil case was considered.

${ }^{3}$ Note that general coordinate invariance is assumed to be a good symmetry on the brane, away from the "boundary" $X^{I}=0$.
} 
A last comment, which is important for our purposes here, concerns the case in which the metric (15) is exact; i.e., it holds to all orders in $\bar{g}_{I z} z$. This is the case where there is no world-sheet tree-level momentum transfer. This naively corresponds to the case of static intersecting branes. However, the whole philosophy of recoil $[6,8]$ implies that, even in that case, there are quantum fluctuations induced by the sum over genera of the world sheet. The latter implies the existence of a statistical distribution of logarithmic deformation couplings of Gaussian type about a mean-field value $\bar{g}_{I z}^{C}=0$. Physically, the couplings $\bar{g}_{I z}$ represent recoil velocities of the intersecting branes; hence these Gaussian fluctuations represent the effects of quantum fluctuations about the zero recoilvelocity case, which may be considered as quantum corrections to the static intersecting-brane case. We therefore consider a statistical average $\ll \cdots \gg$ of the line element (13),

$$
\begin{aligned}
\ll d s^{2} \gg= & -d \phi^{2}+\left(1-\frac{1}{4} \epsilon^{2} \ll \bar{g}_{I z} \bar{g}_{J z} \gg z^{2}\right) d X^{I} d X^{J} \\
& +\left(1+\frac{1}{4} \epsilon^{2} \ll \bar{g}_{I z} \bar{g}_{J z} \gg X^{I} X^{J}\right) d z^{2} \\
& -\epsilon \ll \bar{g}_{I z} \gg z d X^{I} d \phi,
\end{aligned}
$$

where

$$
\ll \cdots \gg=\int_{-\infty}^{+\infty} d \bar{g}_{I z}(\sqrt{\pi} \Gamma)^{-1} e^{-\bar{g}_{I z}^{2} / \Gamma^{2}}(\cdots)
$$

and the width $\Gamma$ has been calculated in [8] and is found after summation over world-sheet genera to be proportional to the string coupling $g_{s}$. In fact, it can be shown [8] that $\Gamma$ scales as $\epsilon \bar{\Gamma}$, where $\bar{\Gamma}$ is independent of $\epsilon$. This will be important later on, when we consider the identification of $\epsilon$ with the target time $t$.

We see from Eq. (17), assuming that $g_{I z}=\left|u_{i}\right|$ where $u_{i}$ $=g_{s} \Delta P_{i} / M_{s}$ is the recoil velocity $[6,8]$, that the average line element $d s^{2}$ becomes

$$
\begin{aligned}
\ll d s^{2} \gg & =-d \phi^{2}+\left(1-\alpha^{2} z^{2}\right)\left(d X^{I}\right)^{2}+\left[1+\alpha^{2}\left(X^{I}\right)^{2}\right] d z^{2}, \\
\alpha & =\frac{1}{2 \sqrt{2}} \epsilon^{2} \bar{\Gamma} .
\end{aligned}
$$

The definition of $\alpha$ comes from evaluating the quantity $\ll \bar{g}_{I z}^{2} \gg$ using the statistical distribution (17). Thus the average over quantum fluctuations leads to a metric of the form (15), but with a parameter $\alpha$ determined by the width (uncertainty) of the pertinent quantum fluctuations [8]. The metric (18) is exact, in contrast to the metric (15) which was derived for $z \ll 1 / \alpha$. However, for our purposes below we shall treat both metrics as exact solutions of some string theory associated with the recoil $[15,16]$.

An important feature of the line element (18) is the existence of a horizon at $z=1 / \alpha$ for Euclidean Neumann coordinates $X^{I}$. Since the Liouville field $\phi$ has decoupled after the averaging procedure, one may consider slices of this field, defined by $\phi=$ const, on which the physics of the observable world can be studied [15]. From a world-sheet renormalization-group view point this slicing procedure corresponds to selecting a specific point in the non-criticalstring theory space. Usually, the infrared fixed point $\phi \rightarrow \infty$ is selected. In that case one considers Eq. (7) a slice for which $\epsilon^{2} \rightarrow 0$. But any other choice could do, so $\alpha$ may be considered a small but arbitrary parameter of our effective theory.

The presence of a horizon raises the issue of how one could analytically continue so as to pass to the space beyond the horizon. The simplest way, compatible [15] with the lowenergy Einstein equations, is to take the absolute value of $1-\alpha^{2} z^{2}$ in the metric element (15) and/or (18). However, we prefer the second approach [16], in which one identifies the (zero mode of the) Liouville mode $\phi$ with the time coordinate $X^{0}$ on the initial $\mathrm{D} p$-brane. In this case, as we shall see, the situation becomes much more interesting, at least in certain regions of the bulk space-time, where one can calculate reliably in a world-sheet perturbative approach. Indeed, far away from the horizon at $|z|=1 / \alpha$, i.e., for $\alpha^{2} z^{2} \ll 1$, the line element corresponding to the space-time (18) becomes, after the identification $\phi=X^{0}$,

$$
d s^{2} \simeq-\alpha^{2} z^{2}\left(d X^{0}\right)^{2}+d z^{2}+\sum_{i=1}^{3}\left(d X^{i}\right)^{2},
$$

implying that $X^{0}$ plays now the role of a Minkowskiansignature temporal variable, despite its original Euclidean nature. This is a result of the identification $\phi=X^{0}$ and the fact that $\phi$ appeared with Minkowskian signature due to the supercriticality (10) of the Liouville string under consideration.

Notice that although the space-time (19) is flat asymptotically as one would expect, and hence satisfies Einstein's equations formally, nevertheless it has a conical singularity when one compactifies the time variable $X^{0}$ on a circle of finite radius corresponding to an inverse "temperature" $\beta$. Formally, this requires a Wick rotation $X^{0} \rightarrow i X^{0}$ and then compactification, $i X^{0}=\beta e^{i \theta}, \quad \theta \in(0,2 \pi]$. The space-time then becomes a conical space-time of the Rindler type,

$$
d s_{\text {conical }}^{2}=\frac{1}{4 \pi^{2}} \alpha^{2} \beta^{2} z^{2}(d \theta)^{2}+d z^{2}+\sum_{i=1}^{3}\left(d X^{i}\right)^{2},
$$

with deficit angle $\delta \equiv 2 \pi-\alpha \beta$. We recall that there is a 'thermalization theorem' for this space-time [17], in the sense that the deficit disappears and the space-time becomes regular, when the temperature is fixed to be

$$
T=\alpha / 2 \pi
$$

The result (21) may be understood physically by the fact that $\alpha$ is essentially related to recoil. As discussed in [9], the problem of considering a suddenly fluctuating (or recoiling) brane at $X^{0}=0$, as in our case above, becomes equivalent to that of an observer in a (non-uniformly) accelerated frame. At times long after the collision the acceleration becomes uniform and equals $\alpha$. This implies the appearance of a non- 


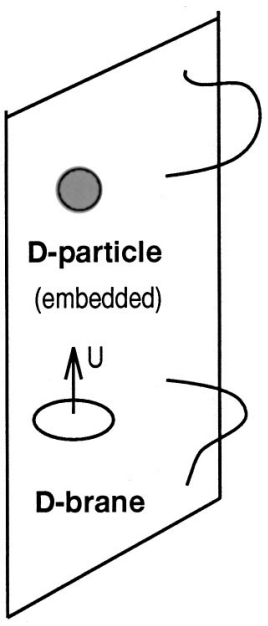

FIG. 1. The world as a D3-brane "punctured" by D particles (D0-branes). The scattering on the D0-brane of string states, either closed (gravitons) or open (matter fields) that reside on the D3brane, cause the D0-brane to recoil, leading to stochastic effects in the propagation of the low-energy states, as well as to non-zero "vacuum" energy on the D3-brane.

trivial vacuum [17], characterized by thermal properties of the form (21). At such a temperature the vacuum becomes just the Minkowski vacuum, while the Unruh vacuum [17] corresponds to $\beta \rightarrow \infty$. Here we have derived this result in a different way than in [9], but the essential physics is the same.

\section{D-PARTICLE RECOIL AND THE DIMENSIONALITY OF THE BRANE WORLD}

In the picture envisaged above, where our world is viewed as a fluctuating D-brane, one may consider more complicated configurations of intersecting branes. The simplest of all cases is the one depicted in Fig. 1, in which a D particle is embedded in a Euclidean D4-brane, which is itself embedded in a higher-dimensional (bulk) space-time.

In this case, any low-energy string state residing on the D3-brane which scatters off the emebedded D0-brane will cause a recoil of the latter and hence a distortion of spacetime, according to the above discussion. The distortion is such as to induce non-trivial contributions to the vacuum energy on the D3-brane, as discussed in detail in $[18,19]$. To see this, we recall that the four-dimensional space-time, in which the defect is embedded, is to be viewed as a bulk space-time from the point of view of the world-sheet approach to the recoil of the D particle. Following the same approach as that leading to Eq. (19), involving the identification of the Liouville field with the target time, $t$, one observes again that there exists an (expanding) horizon, located at $r^{2} \equiv x_{1}^{2}+x_{2}^{2}+x_{3}^{2}=t^{2} / b^{\prime 2}$ where $\left\{x_{i}\right\}, i=1, \ldots, 3$, constitute the bulk dimentions, obeying Dirichlet boundary conditions on the world sheet, and $b^{\prime}$ is related to the momentum uncertainty of the fluctuating $\mathrm{D}$ particle. The variance $b^{\prime}$ was computed [8] using a world-sheet formalism resummed over pinched annuli, which has been argued to be the leadingorder effect for weak string coupling $g_{s}$ :

$$
\left(b^{\prime}\right)^{2}=4 \frac{g_{s}^{2}}{l_{s}^{2}}\left(1-\frac{285}{18} g_{s}^{2} \frac{E_{k i n}}{M_{D} c^{2}}\right)+\mathcal{O}\left(g_{s}^{6}\right)
$$

where $E_{k i n}$ is the kinetic energy scale of the fluctuating (heavy) D particle, $M_{D}=g_{s} / l_{s}$ is the D-particle mass scale, and $l_{s}$ is the string length. Note the dependence of the variance $b^{\prime}$ on the string coupling $g_{s}$, which arises because quantum corrections come from the summation over worldsheet topologies $[16,8]$, and $g_{s}$ is a string-loop counting parameter.

For the region of space-time inside the horizon one obtains the following metric on the D3-brane, as a result of recoil of the $\mathrm{D}$ particle embedded in it:

$$
d s^{2(4)} \simeq \frac{b^{\prime 2} r^{2}}{t^{2}}(d t)^{2}-\sum_{i=1}^{3}\left(d x^{i}\right)^{2}, \quad r^{2}=\sum_{i=1}^{3} x_{i}^{2}<t^{2} / b^{\prime 2} .
$$

Note that the scalar curvature corresponding to the metric (23) has the form $R=-4 / r^{2}$, and as such has a singularity at the initial location $r=0$ of the D-particle defect, as expected.

We can now check whether this metric is a solution of Einstein's equations in a four-dimensional space-time $\left\{x_{i}, t\right\}$, which in our metric and signature conventions the Einstein's equations read

$$
\mathcal{E}_{\mu \nu}=-T_{\mu \nu}
$$

where $T_{\mu \nu}$ is the stress-energy tensor. This is indeed the case provided there exists a four-dimensional dilaton field of the form

$$
\varphi=\ln r+b^{\prime} \ln t
$$

which has non-trivial potential $V(\varphi)$ such that, when combined with field-independent contributions from the vacuum energy $-\Lambda$, one has

$$
\Lambda+V(\varphi)=\frac{2}{r^{2}}
$$

It is important to check that the field $\varphi$ satisfies its classical equations of motion in the space-time (23):

$$
g^{00} \nabla_{0} \partial_{0} \varphi+g^{i j} \nabla_{i} \partial_{j} \varphi=\frac{\delta V(\varphi)}{\delta \varphi}, \quad i=1, \ldots, d .
$$

From Eqs. (27),(26) one obtains the condition

$$
\left.\frac{\delta V(\varphi)}{\delta \varphi}\right|_{\varphi=\varphi_{c}}=-\frac{2}{r^{2}}=-\left[\Lambda+V\left(\varphi_{c}\right)\right]
$$

where $\varphi_{c}$ denotes the configuration (25). From the constraint (28) one then determines $V(\varphi)$ as well as the contributions to the field-independent part of the vacuum energy $-\Lambda$ :

$$
V\left(\varphi_{c}\right)=\frac{1}{r^{2}}, \quad \Lambda=\frac{1}{r^{2}}
$$


in the D3 case considered so far. In the above, we have ignored the fluctuations of the D3-brane in the bulk directions. When these are taken into account, there may be additional contributions $[16,20]$ to the vacuum and excitation energies on the D3-brane, which in fact are time dependent, relaxing to zero asymptotically.

It is interesting to examine whether the metric (23) and the above analysis for the metric equations can be formally extended to $d>3$ bulk (spatial) dimensions. The non-zero components of the Einstein tensor $\mathcal{E}_{\mu \nu}=R_{\mu \nu}-\frac{1}{2} g_{\mu \nu} R$ read, in this general case,

$$
\mathcal{E}_{00}=0, \quad \mathcal{E}_{i j}=-\frac{\delta_{i j}(d-2)}{r^{2}}-\frac{x_{i} x_{j}}{r^{4}}, \quad i, j=1, \ldots, d .
$$

We observe from Eqs. (23),(30) that the metric equations (24) are satisfied for the simple case of a free scalar (dilaton) field $\varphi$ of the form (25), provided $d=3$, independent of the value of $b^{\prime}$. It seems therefore that restoration of the conformal invariance in the case of recoiling $\mathrm{D}$ particles embedded in a $\mathrm{D} p$-brane or, equivalently, the satisfction of the corresponding equations of motion in the Liouville-dressed problem constrains the number of longitudinal dimensions on the $\mathrm{D} p$-brane to 3. In other words, only a D3-brane can intersect with recoiling (fluctuating) D particles in a way consistent with the restoration of conformal invariance in the manner explored here.

\section{ENERGY CONDITIONS AND HORIZONS IN RECOIL- INDUCED SPACE-TIMES}

It is interesting to look at the energy conditions of such space times, which would determine whether ordinary matter can exist within the horizon region displayed above. There are various forms of energy conditions [21], which may be expressed as follows:

$$
\begin{gathered}
\text { strong: }\left(T_{\mu \nu}-\frac{1}{D-2} g_{\mu \nu} T_{\alpha}^{\alpha}\right) \xi^{\mu} \xi^{\nu} \geqslant 0, \\
\text { dominant: } T_{\mu \nu} \xi^{\mu} \eta^{\nu} \geqslant 0, \\
\text { weak: } T_{\mu \nu} \xi^{\mu} \xi^{\nu} \geqslant 0, \\
\text { weaker: } T_{\mu \nu} \zeta^{\mu} \zeta^{\nu} \geqslant 0
\end{gathered}
$$

where $g_{\mu \nu}$ is the metric, $T_{\mu \nu}$ is the stress-energy tensor in a $D$-dimensional space-time, including vacuum-energy contributions, $\xi^{\mu}$ and $\eta^{\mu}$ are arbitrary future-directed time-like or null vectors, and $\zeta^{\mu}$ is an arbitrary null vector. The conditions (31) have been listed in decreasing strength, in the sense that each condition is implied by all its preceding ones.

It can be easily seen from Einstein's equations for the metric (23) that inside the horizon $b^{\prime 2} r^{2} \leqslant t^{2}$ the conditions are satisfied, which implies that stable matter can exist inside such regions of the recoil space-time. On the other hand, outside the horizon the recoil-induced metric assumes the form

$$
d s^{2(4)} \simeq\left(2-\frac{b^{\prime 2} r^{2}}{t^{2}}\right)(d t)^{2}-\sum_{i=1}^{3}\left(d x^{i}\right)^{2}, \quad r^{2}>t^{2} / b^{\prime 2}
$$

The induced scalar curvature is easily found to be

$$
R=-4 b^{\prime 2}\left(-3 t^{2}+b^{\prime 2} r^{2}\right) /\left(-2 t^{2}+b^{\prime 2} r^{2}\right)^{2} .
$$

Notice that there is a curvature singularity at $2 t^{2}=b^{\prime 2} r^{2}$, which is precisely the point where there is a signature change in the metric (32).

Notice also that, in order to ensure a Minkowskian signature in the space-time (32), one should impose the restriction

$$
2>\frac{b^{\prime 2} r^{2}}{t^{2}}>1
$$

Outside this region, the metric becomes Euclidean, which matches our formal initial construction with a static Euclidean D4-brane embedded in a higher-dimensional bulk spacetime. Notice that, in such a region, one can formally pass to a Minkowskian four-dimensional space-time by making a Wick rotation of the Euclidean time coordinate $X^{0}$. In this Wick-rotated framework, the space-time inside the bubbles retains its Minkowskian signature due to the specific form of the metric (23).

The above metric (32) does not satisfy simple Einstein's equations, but this was to be expected, since the formation of such space-times is not necessarily a classical phenomenon. ${ }^{4}$ Below, we link this fact with the failure of the energy conditions in this exterior geometry.

It can easily be shown that the weaker energy condition (31) can be satisfied for times $t$ such that

$$
\frac{b^{\prime 2} r^{2}}{t^{2}} \simeq 1+\varepsilon, \quad \varepsilon \rightarrow 0^{+},
$$

i.e., on the initial horizon. To see this, it suffices to notice that the weaker energy condition reads, in this case,

$$
\left(4 t^{2}-b^{\prime 2} r^{2}\right)\left(2-\frac{b^{\prime 2} r^{2}}{t^{2}}\right)\left(\zeta^{0}\right)^{2} \leqslant b^{\prime 2}\left(\sum_{i=1}^{3} x^{i} \zeta^{i}\right)^{2}
$$

where we used the fact that $\zeta^{\mu}$ is a null vector. Choosing $\zeta_{1} \neq 0, \quad \zeta_{i}=0, \quad i=2,3$, it can be shown that the right-hand side of the above inequality can be bounded from above by

$$
b^{\prime 2} r^{2} \sum_{i=1}^{3}\left(\zeta^{i}\right)^{2}=b^{\prime 2} r^{2}\left(2-\frac{b^{\prime 2} r^{2}}{t^{2}}\right)\left(\zeta^{0}\right)^{2}
$$

\footnotetext{
${ }^{4}$ On the other hand, the satisfaction of conformal invariance conditions on a resummed world sheet, as a result of Liouville dressing, implies general "Liouville equations" in which the $\beta$ functions and the central charge deficit $Q$ incorporate higher-genus world-sheet (resummed) effects. Unfortunately, general expressions for these objects are currently beyond our calculational reach.
} 


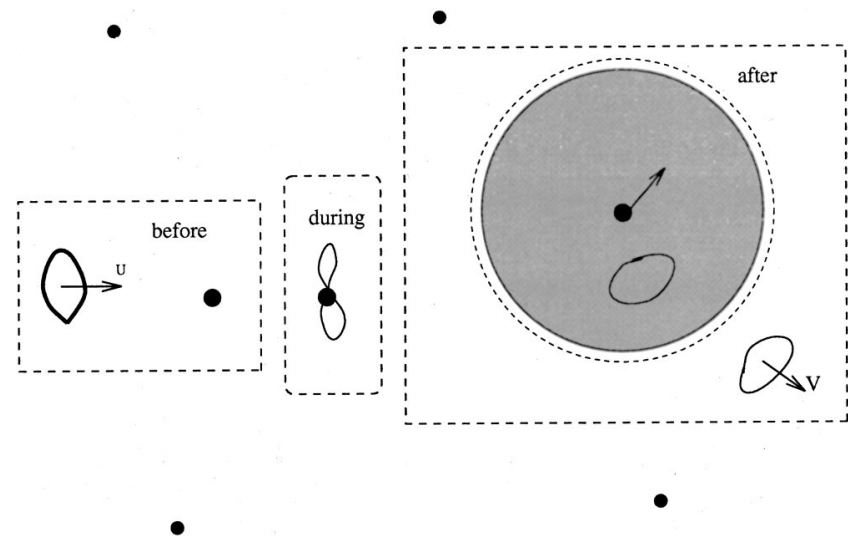

FIG. 2. A schematic representation of scattering in D-foam background. The dashed boxes represent events just before, during and after the scattering of a closed-string probe on one particular D-brane defect. The scattering results in the formation of a shaded bubble, expanding as indicated by the dotted line, inside which matter can be trapped and there is an energy-dependent refractive index.

which, on account of the requirement (35), would imply $\left(2 t^{2}-b^{\prime 2} r^{2}\right) \leqslant 0$. This is in contradiction with the range of validity of Eq. (32), unless one lies on the initial horizon (34). Notice that in this region of space-time there is a smooth matching between the interior (23) and the exterior (32) geometries. In such regions of space-time, surrounding the recoiling defect, matter can exist in a stable form.

The above considerations suggest that matter can be trapped inside such horizon regions around a fluctuating D-particle defect. This sort of trapping is interesting for our space-time-foam picture, as it implies that such microscopic D-brane horizons act in a similar way as the intuitive description of a macroscopic black-hole horizon discussed in the Introduction, Eq. (1), as illustrated in Fig. 2.

To reinforce the interpretation that matter is trapped in the interior of a region described by the metric (23), we now show that a matter probe inside the horizon "bubble" experiences an energy-dependent velocity of light. First rewrite the metric in a Friedmann-Robertson Walker (FRW) form

$$
d s^{2}=e^{2 \ln r}\left(b^{\prime 2} d t_{F R W}^{2}-\frac{1}{r^{2}} \sum_{i=1}^{3}\left(d x_{i}\right)^{2}\right)
$$

where we were careful when performing coordinate redefinitions not to absorb in them the factor $b^{\prime}$, which, depends Eq. (22) on the energy scale of the matter probe. We are interested in matter at various energies propagating simultaneously in such a space-time, and performing a coordinate transformation could not absorb an energy-dependent factor such as $b^{\prime}$. When we consider the encounter of a matter probe, such as a photon, with a fluctuating D-particle defect, the kinetic-energy scale $E_{k i n}$ may be identified with the energy scale $E$ of the matter probe. We recall that energy con- servation has been proved rigorously in the world-sheet approach to D-brane recoil [8], and survives the resummation over higher genera.

We observe from Eq. (36) that the overall scale factor may be absorbed into a redefinition of the spatial part of the dilaton (25), implying that stable matter experiences an energy-dependent "light velocity"

$$
c_{\text {int }}(E)=b^{\prime} c=2 c g_{s}\left(1-\frac{285 g_{s}^{2} E}{18 M_{D} c^{2}}\right)^{1 / 2}
$$

in the space-time (23), where $M_{D}=M_{s} / g_{s}$ is the D-particle mass scale. The energy-independent factor $2 g_{s}$ may in fact be absorbed into the normalization of the FRW time coordinate $t_{F R W}$, thereby making a smooth connection with the velocity of light in vacuo in the limiting case of $E / M_{D} c^{2}$ $\rightarrow 0$. It is important to note that, because of the specific form $E q$. (22) of the variance $b^{\prime}$, the resulting effective velocity (37) in the interior of the bubble is subluminal [22]. On the other hand, we see from Eq. (32) that matter propagates at the normal in vacuo light velocity $c$ in the exterior part of the geometry.

If one considers pulses containing many photons of different energies $[23,24]$, then the various photons will experience, as a result of the dynamical formation of horizons, changes in their mean effective velocities corresponding on average to a refractive index $\Delta c(E)$, where the effective light velocity

$$
c(E)=c\left(1-\xi \frac{g_{s} E}{M_{s} c^{2}}\right)
$$

Here $\xi$ is a quantity that depends on the actual details of the scenario for quantum space-time foam, in particular on the density of the D-brane defects in space. In a dilute-gas approximation, $\xi$ might plausibly be assumed to be of order 1, as can be seen as follows. Consider a path $L$ of a photon, which encounters $\mathcal{N}$ fluctuating D-particle defects. Each defect creates a bubble which is expected to be close to the Planckian size $l_{s}$, for any reasonable model of space-time foam. Inside each bubble, the photon propagates with velocity (37), whereas outside it propagates with the velocity of light in vacuo $c$. The total time of flight for this probe will therefore be given by

$$
t_{\text {total }}=\frac{L-\mathcal{N} l_{s}}{c}+\mathcal{N} \frac{l_{s}}{c}\left(1-g_{s}^{2} \frac{285}{18} \frac{E}{M_{D} c^{2}}\right)^{-1 / 2} .
$$

In a "dilute gas approximation" for the description of spacetime foam, it is natural to assume that a photon encounters, on average, an $\mathcal{O}(1)$ D-particle defect in each Planckian length $l_{s}$, so that $\mathcal{N} \sim \xi L / l_{s}$, where $\xi \leqslant 1$. From Eq. (39), then, one obtains a delay in the arrival time of a photon of order 


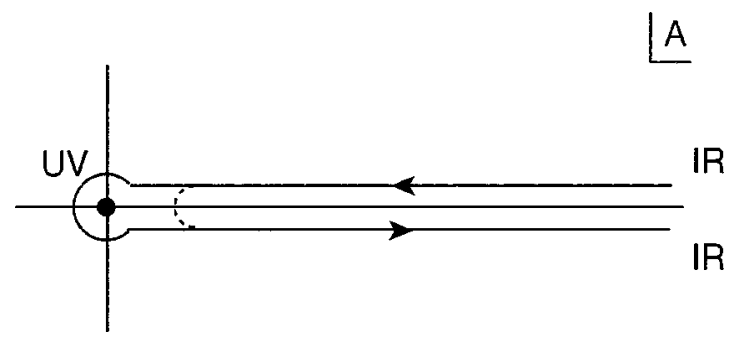

FIG. 3. Contour of integration appearing in the analytically continued (regularized) version of world-sheet Liouville string correlators. The quantity $A$ denotes the (complex) world-sheet area. This is known in the literature as the Saalschutz contour, and has been used in conventional quantum field theory to relate dimensional regularization to the Bogoliubov-Parasiuk-Hepp-Zimmermann renormalization method. Upon the identification of the Liouville field with target time [16], this curve resembles closed-time paths in nonequilibrium field theories [26].

$$
\Delta t \sim \xi g_{s}^{2} \frac{285}{36} \frac{L E}{M_{D} c^{3}}+\cdots
$$

corresponding to the effective velocity $(38) .^{5}$

\section{BREATHING HORIZONS IN LIOUVILLE STRING THEORY}

The tendency of the horizon (34) to expand is a classical feature. Upon quantization, which corresponds in our picture to a proper resummation over world-sheet topologies, one expects a phenomenon similar to Hawking radiation. Such a phenomenon would decelerate and stop the expansion, leading eventually to the shrinking of the horizon. This would be a dynamical picture of space-time foam, which unfortunately at present is not fully available, given that at microscopic distances the world-sheet perturbative analysis breaks down. However, we believe that this picture is quite plausible, and we can support these considerations formally by recalling that time $t$ is the Liouville field in our formalism.

As we have pointed out previously, the dynamics of the Liouville field exhibits a "bounce', behavior, when considered from a world-sheet view point $[25,16]$, as illustrated in Figs. 3 and 4. This is a general feature of non-critical strings, whenever the Liouville field is viewed as a local renormalization-group scale of the world sheet. As emphasized in [19], the bounce picture is necessitated by the decomposition of the Liouville world-sheet correlators as ordinary scattering-matrix elements in target space. Specifically, these correlators diverge with the world-sheet area scale $A$ in the infrared limit $A \rightarrow 0^{+}$. One may regularize such divegences by defining the world-sheet path integral over the Liouville mode on the analytically continued curve illus-

\footnotetext{
${ }^{5}$ In conventional string theory, $g_{s}^{2} / 2 \pi \sim 1 / 20$, and the overall numerical factor in Eq. (40) is of order $4.4 \xi$. However, $g_{s}$ should rather be considered an arbitrary parameter of the model, which may then be constrained by phenomenological observations [24] through limits on Eq. (40).
}

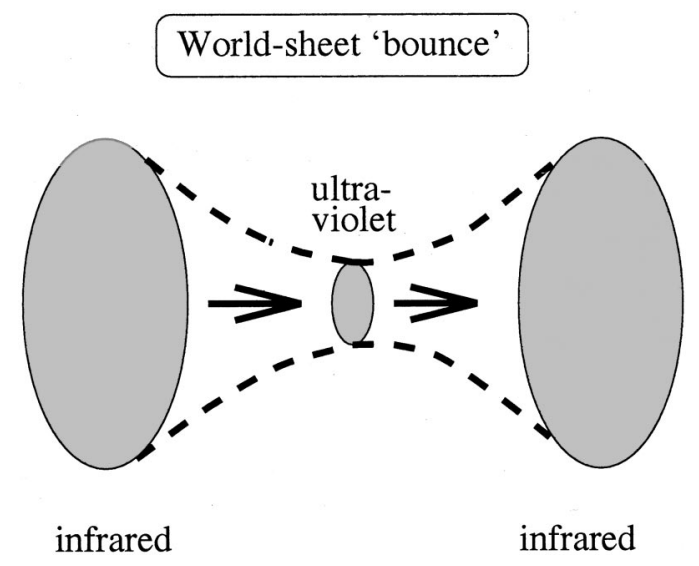

FIG. 4. The evolution of the world sheet of the Liouville string, when the Liouville mode lies on the (dashed) curve of Fig. 3. Upon interpreting the (zero-mode of the) Liouville field as time, the above "bounce"' evolution suggests a 'breathing mode' for the Liouville universe, which from our point of view represents a formal description of a space-time foam "bubble."

trated in Fig. 3. When one inteprets the (zero mode of the) Liouville field $\phi$ as time [16], $t \propto \log A$; therefore, the contour of Fig. 3 represents evolution in time, as seen in Fig. 4, in both directions between fixed points of the world-sheet renormalization group: infrared fixed point $\rightarrow$ ultraviolet fixed point $\rightarrow$ infrared fixed point.

When one integrates over the Saalschultz contour in Fig. 3 , the integration around the simple pole at $A=0$ yields an imaginary part $[25,16]$, associated with the instability of the Liouville vacuum. We note, on the other hand, that the integral around the dashed contour shown in Fig. 3, which does not encircle the pole at $A=0$, is well defined. This can be intepreted as a well-defined $S$-matrix element, which is not, however, factorizable into a product of $S$ - and $S^{\dagger}$-matrix elements, due to the $t$ dependence acquired after the identification $t=-\log A .^{6}$ The absence of factorization is linked to the evolution (1) from a pure state $|A, B\rangle$ to a mixed density matrix (2), which cannot be described by a conventional $S$ matrix.

In our approach, the logarithmic algebra of the recoil operators forces the regularizing parameter $\epsilon$, Eq. (7), to be identified with the logarithm of the world-sheet area scale $A=|L / a|^{2}$ and, hence, with the target time. In the bounce picture outlined above, there will be a 'breathing mode' in the recoil-induced space-time, characterized by two directions of time, corresponding to the processes of expansion, stasis and shrinking of the horizon in the recoil-induced space-time (23), all within a few Planckian times. This is the Liouville-string description of Hawking radiation.

\section{OUTLOOK}

We have discussed in this article a microscopic mechanism for the dynamical formation of horizons by the colli-

\footnotetext{
${ }^{6}$ This formalism is similar to the closed-time-path (CTP) formalism used in non-equilibrium quantum field theories [26].
} 
sions of closed-string particle "probes" with recoiling D-particle defects embedded in a $p$-dimensional space time, which may in turn be viewed as a $\mathrm{D} p$ brane domain wall in a higher-dimensional target space. As we have argued before, the correct incorporation of recoil effects, which are unavoidable in any quantum theory of gravity that reproduces the conceptual framework of general relativity in the classical limit, necessitates a Liouville string approach in the context of a (perturbative) world-sheet framework.

The most important result of our approach in this paper is the demonstration of the dynamical formation of breathing horizons, which follows directly from the restoration of conformal invariance by means of Liouville dressing. The horizon regions were discovered using the positive-energy theorems for the recoil-induced space-time. We have been able to show that such regions form bubbles with a non-trivial refractive index, with light propagation that is always subluminal [22], because of the specific properties of the space-time induced by our treatment of D-particle recoil. The breathing nature of the horizons, which follows from specific properties of the Liouville dynamics, is the best candidate we have in this framework for a quantum space-time foam, generalizing appropriately the Hawking radiation of conventional macroscopic black holes to the microscopic D-brane case.

The non-trivial optical properties induced by the propagation of light in such a fluctuating space-time may be subject to experimental verification in the foreseeable future, and are already constrained by existing data [23,24]. The fact that the refractive index in the bubbles of space-time foam is subluminal implies the absence of birefringence in light propagation, which is, however, possible in other approaches to space-time foam [27].

One curiosity of our analysis has been that the requirement of restoring conformal invariance by means of the Liouville field, which in our approach is identified with the target time, is quite restrictive. It implies within this ap- proach that only D3-branes can intersect consistently with fluctuating D particles. The result is surprising, as it seems to provide a mathematical reason for the fact that we live in four dimensions only. However, the model is oversimplified, in the sense that only dilaton and graviton fields have been considered so far in modelling the dynamics of the distortion of the space-time due to the fluctuating defect. This may in some sense be analogous to the way the critical dimension of conventional string theories was revealed in the context of a $\sigma$-model approach, when only the conformal anomaly contributions in a flat target space were considered. However, there have been many independent confirmations of the critical dimension in this "traditional" string case, coming, e.g., from the no-ghost theorems and the closure of the Lorentz algebra. In the present case, we currently lack further support of our result from an independent calculation, but we consider it as worthy of further investigation.

Leaving aside this issue of the critical dimension, our theoretical model is admittedly crude, and should by no means be considered as complete. However, we believe that it provides a concrete example how space-time foam might arise in the context of modern string-D-brane theory. Certainly much more work, both theoretical and "phenomenological," is necessary before even tentative conclusions are reached on these important matters. But it is our firm belief that the model presented here contains the seeds for an eventual understanding of many important issues associated with the space-time foam structure of quantum gravity, and for this reason it deserves further and more detailed studies before it can be excluded.

\section{ACKNOWLEDGMENTS}

The work of N.E.M. is partially supported by PPARC (UK). That of D.V.N. is partially supported by DOE grant DE-F-G03-95-ER-40917. N.E.M. and D.V.N. also thank H. Hofer for his interest and support.
[1] J. Polchinski, Phys. Rev. Lett. 75, 4724 (1995).

[2] J. Ellis, N. E. Mavromatos, and D. V. Nanopoulos, Phys. Lett. B 284, 27 (1992); 284, 43 (1992).

[3] A. Sen, invited talk at the 29th International Conference on High-Energy Physics (ICHEP 98), Vancouver, Canada, 1998, hep-ph/9810356.

[4] S. W. Hawking, Commun. Math. Phys. 43, 199 (1975).

[5] J. A. Wheeler, Ann. Phys. (N.Y.) 2, 605 (1957); S. W. Hawking, Nucl. Phys. B144, 349 (1978); S. W. Hawking, D. N. Page, and C. N. Pope, Phys. Lett. 86B, 175 (1979); J. Ellis, J. Hagelin, D. Nanopoulos, and M. Srednicki, Nucl. Phys. B241, 381 (1984); T. Banks, L. Susskind, and M. E. Peskin, ibid. B244, 125 (1984); L. J. Garay, Phys. Rev. D 58, 124015 (1998).

[6] I. I. Kogan, N. E. Mavromatos, and J. F. Wheater, Phys. Lett. B 387, 483 (1996).

[7] J. Ellis, N. E. Mavromatos, and D. V. Nanopoulos, Int. J. Mod. Phys. A 13, 1059 (1998).

[8] N. E. Mavromatos and R. J. Szabo, Phys. Rev. D 59, 104018 (1999).
[9] J. Ellis, P. Kanti, N. E. Mavromatos, D. V. Nanopoulos, and E. Winstanley, Mod. Phys. Lett. A 13, 303 (1998).

[10] V. Gurarie, Nucl. Phys. B410, 535 (1993); J. S. Caux, I. I. Kogan, and A. M. Tsvelik, ibid. B466, 444 (1996); M. A. I. Flohr, Int. J. Mod. Phys. A 11, 4147 (1996); M. R. Gaberdiel and H. G. Kausch, Nucl. Phys. B489, 293 (1996); I. I. Kogan and N. E. Mavromatos, Phys. Lett. B 375, 111 (1996); M. R. Rahimi-Tabar, A. Aghamohammadi, and M. Khorrami, Nucl. Phys. B497, 555 (1997); I. I. Kogan, A. Lewis, and O. A. Soloviev, Int. J. Mod. Phys. A 13, 1345 (1998).

[11] F. David, Mod. Phys. Lett. A 3, 1651 (1988).

[12] J. Distler and H. Kawai, Nucl. Phys. B321, 509 (1989).

[13] J. Ellis, N. E. Mavromatos, and E. Winstanley, Phys. Lett. B 476, 165 (2000).

[14] I. Antoniadis, C. Bachas, J. Ellis, and D. V. Nanopoulos, Nucl. Phys. B328, 117 (1989).

[15] G. K. Leontaris and N. E. Mavromatos, Phys. Rev. D 61, 124004 (2000).

[16] For reviews, see J. Ellis, N. E. Mavromatos, and D. V. Nan- 
opoulos, Chaos Solitons Fractals 10, 345 (1999); J. Ellis, N. E. Mavromatos, and D. V. Nanopoulos, invited contribution to the 2nd International Conference on Physics Beyond the Standard Model: Beyond the Desert 99: Accelerator, Nonaccelerator and Space Approaches, Ringberg Castle, Tegernsee, Germany, 1999, gr-qc/9909085; J. Ellis, N. E. Mavromatos, and D. V. Nanopoulos, presented at 4th International Symposium on Sources and Detection of Dark Matter in the Universe (DM 2000), Marina del Rey, California, 2000, gr-qc/0005100.

[17] W. G. Unruh, Phys. Rev. D 14, 870 (1976).

[18] J. Ellis, N. E. Mavromatos, and D. V. Nanopoulos, gr-qc/9810086.

[19] J. Ellis, N. E. Mavromatos, and D. V. Nanopoulos, Int. J. Mod. Phys. A 13, 5093 (1998).

[20] A. Campbell-Smith and N. E. Mavromatos, Phys. Lett. B (to be published), hep-th/0003262.

[21] R. M. Wald, General Relativity (University of Chicago Press,
Chicago, 1984).

[22] J. Ellis, N. E. Mavromatos, and D. V. Nanopoulos, Phys. Rev. D 61, 027503 (2000).

[23] G. Amelino-Camelia, J. Ellis, N. E. Mavromatos, D. V. Nanopoulos, and S. Sarkar, Nature (London) 393, 763 (1998); see also S. D. Biller et al., Phys. Rev. Lett. 83, 2108 (1999); P. Kaaret, Astron. Astrophys. 345, L3 (1999).

[24] J. Ellis, K. Farakos, N. E. Mavromatos, V. A. Mitsou, and D. V. Nanopoulos, Astrophys. J. 535, 139 (2000).

[25] I. Kogan, Phys. Lett. B 265, 269 (1991).

[26] J. Schwinger, J. Math. Phys. 2, 407 (1961); H. Umezawa, Advanced Field Theory: Micro, Macro and Thermal Concepts (AIP, New York, 1993). For recent discussions, see E. Calzetta and B. L. Hu, Phys. Rev. D 37, 2878 (1988); E. Calzetta, S. Habib, and B. L. Hu, ibid. 37, 2901 (1988).

[27] R. Gambini and J. Pullin, Phys. Rev. D 59, 124021 (1999). 